\title{
AN AUTOMATIC SCREENING METHOD TO DETECT OPTIC DISC IN THE RETINA
}

\author{
Murugan.R ${ }^{1}$, Dr.Reeba Korah ${ }^{2}$ \\ ${ }^{1}$ Research Scholar, Centre for Research, Anna University of Technology Chennai \\ murugan.rmnegmail.com \\ ${ }^{2}$ Professor, St.Joseph's College of Engineering, Chennai \\ reeba26in@yahoo.co.in
}

\begin{abstract}
The location of Optic Disc (OD) is of critical importance in retinal image analysis. This research paper carries out a new automated methodology to detect the optic disc (OD) in retinal images. OD detection helps the ophthalmologists to find whether the patient is affected by diabetic retinopathy or not. The proposed technique is to use line operator which gives higher percentage of detection than the already existing methods. The purpose of this project is to automatically detect the position of the OD in digital retinal fundus images. The method starts with converting the RGB image input into its LAB component. This image is smoothed using bilateral smoothing filter. Further, filtering is carried out using line operator. After which gray orientation and binary map orientation is carried out and then with the use of the resulting maximum image variation the area of the presence of the $O D$ is found. The portions other than $O D$ are blurred using $2 D$ circular convolution. On applying mathematical steps like peak classification, concentric circles design and image difference calculation, OD is detected. The proposed method was evaluated using a subset of the STARE project's dataset and the success percentage was found to be $96 \%$.
\end{abstract}

\section{KEY WORDS}

Automated detection, Optic Disc, Line Filter Operator, Retinal Imaging.

\section{INTRODUCTION}

In ophthalmology, the automatic detection of optic disc may be of considerable interest for computer assisted diagnosis. Detecting and counting lesions in the human retina like microaneurysms and exudates is a time consuming task for ophthalmologists and prone to human error. That is why much effort has been done to detect lesions in the human retina automatically. Finding the main components in the fundus images helps in characterizing detected lesions and in identifying false positives. The detection of the optic disc is the first step in the early detection of the diabetic retinopathy.

This paper presents a line operator that is designed to locate the OD from retinal images accurately. Our proposed line operator is designed to capture the circular brightness structure associated with the OD. In particular, it evaluates the image variation along multiple oriented line segments and locates the OD based on the orientation of the line segment with the maximum/minimum variation. Fig. 1 (a \& b) shows an example of retinal image in digital retinal images for vessel extraction (DRIVE) project's dataset. 
International Journal of Advanced Information Technology (IJAIT) Vol. 2, No.4, August 2012

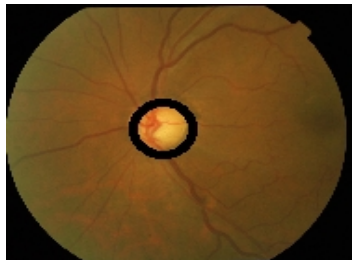

(a)

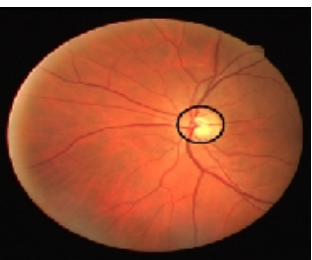

(b)

Fig. 1. Circular brightness structure associated with the OD. (a \& b) Example of retinal image in DRIVE project's dataset with OD labelled by a bold black circle.

The proposed method has several advantages. First, the designed line operator is tolerant to the retinal lesion and various types of imaging artifacts that most image-characteristics-based methods cannot handle properly. The tolerance to the imaging artifacts and retinal lesion can be explained by the proposed line operator that is designed to capture the unique circular brightness structure associated with the OD. Second, the designed line operator is stable and easy for implementation. It requires neither the retinal blood vessel nor the macula information.

The remaining part of the paper is organized as follows. Most of the available methods for automatic OD detection are reviewed in Section II. Section III presents the proposed algorithm. The results are presented and discussed in Sections IV. Finally, conclusion and further work are found in Section V.

\section{OD DETECTION METHODS : A LITERATURE REVIEW}

Automatic optic disc (OD) detection from retinal images is a very important task in ocular image analysis [1], [2] and computer-aided diagnosis of various types of eye diseases [3]-[5]. It is often a key step for the detection of other anatomical retinal structures, such as retinal blood vessels and macula [1], [6], [7], [8]. More importantly, it helps to establish a retinal coordinate system that can be used to determine the position of other retinal abnormalities, such as exudates, drusen, and hemorrhages [9], [10].

Some OD detection techniques have been reported in the literature. The early techniques make use of different types of OD specific image characteristics. In particular, some techniques search for the brightest regions [11], [12] or regions with the highest image variation [13], [14] resulting from the bright OD and the dark blood vessels within the OD. The limitation of these methods is that many retinal images suffer from various types of retinal lesion, such as drusen, exudates, microaneurysms, and hemorrhage, and imaging artifacts, such as haze, lashes, and uneven illumination (as illustrated in Figs. 9 and 10) that often produce brighter regions or regions with higher image variation compared to the OD.

Several OD detection techniques make use of anatomical structures among the OD, macula, and retinal blood vessels. For example, some methods are based on the anatomical structure that all major retinal blood vessels radiate from the OD [15]-[18]. Some other methods make use of the relative position between the OD and the macula that often varies within a small range [19], [20]. Compared with the image characteristics, the anatomical structures are more reliable under the presence of retinal lesion and imaging artifacts. However, the extraction of either retinal blood vessels or the macula is often a nontrivial task by itself.

Line operators have been used to locate linear structures from different types of images. For example, Zwiggelaar et al. used a line operator to detect linear structures from mammographic images [21], where a line strength is evaluated by the difference between the largest average image intensity along one oriented line segment and the average image intensity within a local 
International Journal of Advanced Information Technology (IJAIT) Vol. 2, No.4, August 2012

neighbourhood window. Ricci and Perfetti [22] used a similar line operator to detect the linear structures that are associated with the retinal blood vessels.

\section{PROPOSED METHOD}

This section presents the proposed OD detection technique. In particular, we divide this section into four subsections, which deal with the retinal image pre-processing, designed line operator, OD detection, and discussion, respectively.

\subsection{Retinal Image Pre-processing}

Retinal images need to be pre-processed before the OD detection. As the proposed technique makes use of the circular brightness structure of the OD, the lightness component of a retinal image is first extracted. We use the lightness component within the LAB color space, where the OD detection usually performs the best [23]. For the retinal image in Fig. 1(a), Fig. 2(a) shows the corresponding lightness image.

The retinal image is then smoothed to enhance the circular brightness structure associated with the OD. We use a bilateral smoothing filter [24] that combines geometric closeness and photometric similarity as follows:

$h(x)=k^{-1}(x) \int_{-\infty}^{\infty} \int_{-\infty}^{\infty} f(\xi) c(\xi, x) s(f(\xi) ; f(x)) d \xi$

With the normalization factor

$\mathrm{k}(\mathrm{x})=\int_{-\infty}^{\infty} \int_{-\infty}^{\infty} \mathrm{c}(\xi, \mathrm{x}) \mathrm{s}(\mathrm{f}(\xi) ; \mathrm{f}(\mathrm{x})) \mathrm{d} \xi$

where $\mathrm{f}(\mathrm{x})$ denotes the retinal image under study. $\mathrm{c}(\xi, \mathrm{x})$ and $\mathrm{s}(\mathrm{f}(\xi), \mathrm{f}(\mathrm{x}))$ measure the geometric closeness and the photometric similarity between the neighborhood center $\mathrm{x}$ and a nearby point $\xi$. We set both $\mathrm{c}(\xi, \mathrm{x})$ and $\mathrm{s}(\mathrm{f}(\xi), \mathrm{f}(\mathrm{x}))$ by Gaussian functions. The geometric spread $\sigma_{d}$ and the photometric spread $\sigma_{r}$ of the two Gaussian functions are typically set at 10 and 1 as reported in [24]. For the retinal image in Fig. 2(a), Fig. 2(b) shows the filtered retinal image.

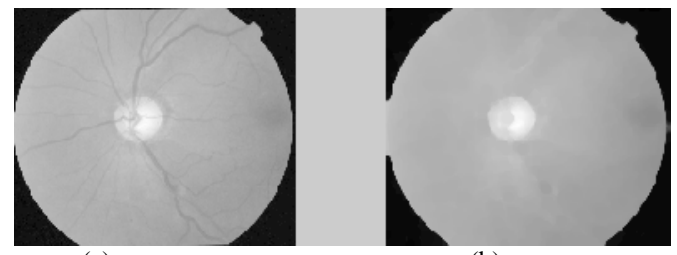

Fig. 2. Retinal image pre-processing. (a) Lightness of the example retinal image in LAB color space. (b) Enhanced retinal image by bilateral smoothing where multiple crosses along a circle label the pixels to be used to illustrate the image variation along multiple oriented line segments.

\subsection{Designed Line Operator}

A line operator is designed to detect circular regions that have similar brightness structure as the OD. For each image pixel at $(\mathrm{x}, \mathrm{y})$, the line operator first determines $\mathrm{n}$ line segments $\mathrm{L}_{i}, \mathrm{i}$ $=1 \ldots \mathrm{n}$ of specific length $\mathrm{p}$ (i.e., number of pixels) at multiple specific orientations that center at 
International Journal of Advanced Information Technology (IJAIT) Vol. 2, No.4, August 2012

$(\mathrm{x}, \mathrm{y})$. The image intensity along all oriented line segments can thus be denoted by a matrix I(x, y) ${ }_{n \times p}$, where each matrix row stores the intensity of $\mathrm{p}$ image pixels along one specific line segment. The line operator that uses 20 oriented line segments and sets the line length $p=21$, each line segment $\mathrm{L}_{i}$ at one specific orientation can be divided into two line segments $\mathrm{L}_{i} \quad, 1$ and $\mathrm{L}_{i} \quad, 2$ of the same length $(\mathrm{p}-1) / 2$ by the image pixel under study (i.e., the black cell). The image variation along the oriented line segments can be estimated as follows:

$D_{i} \quad(x, y)=\left\|f_{m d n} \quad\left(I L_{i, 1} \quad(x, y)\right)-f_{m d n}\left(I L_{i, 2} \quad(x, y)\right)\right\|, i=1 \ldots \ldots . . n$

Where $\mathrm{f}_{m d n}(\cdot)$ denotes a median function. $\mathrm{f}_{m d n}\left(\operatorname{IL}_{i, 1} \quad(\mathrm{x}, \mathrm{y})\right)$ and $\mathrm{f}_{m d n} \quad\left(\operatorname{IL}_{i, 2} \quad(\mathrm{x}, \mathrm{y})\right)$

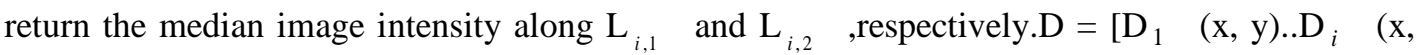
$\left.\mathrm{y}) \ldots \mathrm{D}_{n}(\mathrm{x}, \mathrm{y})\right]$ is, therefore, a vector of dimension $\mathrm{n}$ that stores the image variations along $\mathrm{n}$ oriented line segments.

The orientation of the line segment with the maximum/minimum variation has specific pattern that can be used to locate the OD accurately. For retinal image pixels, which are far away from the $\mathrm{OD}$, the orientation of the line segment with the maximum/minimum variation is usually arbitrary, but for those around the OD, the image variation along Lc [labeled in Fig. 1(b)] usually reach the maximum, whereas that along Lt reaches the minimum. Fig. 4 shows the image variation vectors $\mathrm{D}(\mathrm{x}, \mathrm{y})$ of eight pixels that are labeled by crosses along a circle shown in Fig. 2(b). Suppose that there is a Cartesian coordinate system centered at the OD, as shown in Fig. 2(b). For the retinal image pixels in quadrants I and III, the image variations along the 1st-10th [i.e., Lt in Fig. 1(b)] and the 11th-20th (i.e., Lc ) line segments labeled in Fig. 3 reach the minimum and the maximum, respectively, as shown in Fig. 4. But for the retinal image pixels in quadrants II and IV, the image variations along the 1st-10th and the 11th-20th line segments instead reach the maximum and the minimum, respectively.

An orientation map can, therefore, be constructed based on the orientation of the line segment with the maximum (or minimum) variation as follows:

$\mathrm{O}(\mathrm{x}, \mathrm{y})=\operatorname{argmax} \mathrm{D}(\mathrm{x}, \mathrm{y})$

where $\mathrm{D}(\mathrm{x}, \mathrm{y})$ denotes the image variation vector evaluated in (3). In addition, a binary orientation map can also be constructed by classifying the orientation of the line segment with the maximum variation into two categories as follows:

$$
Q(x, y)=\left\{\begin{array}{c}
1, \text { if } \operatorname{argmax} D(x, y)<\frac{n}{2}+1 \\
-1, \text { Otherwise }
\end{array}\right.
$$

where $\mathrm{n}$ refers to the number of the oriented line segments used in the line operator. 


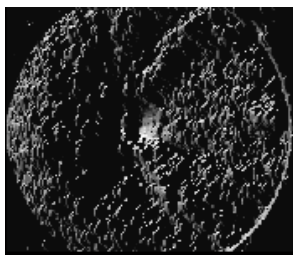

(a)

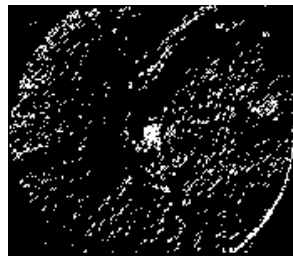

(b)

Fig. 3. Orientation map of the retinal image in Fig. 2(b). (a) Gray orientation map that is determined by using (4). (b) Binary orientation map that is determined by using (5).

For the retinal image in Fig. 1(a), Fig. 3(a) and (b) shows the determined gray orientation map and binary orientation map, respectively. As shown in Fig. 3(a), for retinal image pixels in quadrants I and III around the OD, the orientation map is a bit dark because the orientation of the line segment with the maximum variation usually lies between 1 and $(n / 2)+1$. However, for retinal image pixels in quadrants II and IV, the orientation map is bright because the orientation of the line segment with the maximum variation usually lies between $\mathrm{n} / 2$ and $\mathrm{n}$. The binary orientation map in Fig. 3(b) further verifies such orientation pattern. The OD will then be located by using the orientation map to be described in the following.

\subsection{OD Detection}

We use a line operator of 20 oriented line segments because line operators with more line segments have little effect on the orientation map. The line length $p$ is set as follows:

$p=k R$

where $\mathrm{R}$ denote the radius of the central circular region of retinal images as illustrated in Fig. 1(a). Parameter $\mathrm{k}$ controls the line length, which usually lies between $1 / 10$ and $1 / 5$ based on the relative OD size within retinal images [25]. The use of $\mathrm{R}$ incorporates possible variations of the image resolution.

The specific pattern within the orientation map is captured by a 2-D circular convolution mask shown at the upper left corner of two peak images in Fig. 4.b. As shown in Fig. 4, the convolution mask can be divided into four quadrants, where the cells within quadrants I and III are set at-1, whereas those within quadrants II and IV are set at 1 based on the specific pattern within the orientation map. An orientation map can thus be converted into a peak image as follows:

$\mathrm{P}(\mathrm{x}, \mathrm{y})=\sum_{x=x_{0}-m}^{x_{0}+m} \sum_{y=y_{0}-m}^{y_{0}+m} M(x, y) O(x, y)$

where $\left(\mathrm{x}_{0} \mathrm{y}_{0}\right)$ denotes the position of the retinal image pixel under study. $\mathrm{M}(\mathrm{x}, \mathrm{y})$ and $\mathrm{O}(\mathrm{x}, \mathrm{y})$ refer to the value of the convolution mask and the orientation map at (x,y), respectively. Parameter $m$ denotes the radius of the circular convolution mask that can be similarly set as $p$. 

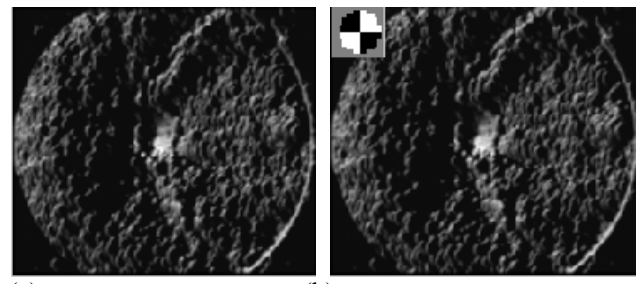

(a)

Fig. 4. Peak images determined by a 2-D circular convolution mask shown in the upper left corner. (a) Peak image produced through the convolution of the gray orientation map in Fig. 3(a). (b) Peak image produced through the convolution of the binary orientation map in Fig. 3(b).

For the orientation maps in Fig. 5(a) and (b), Fig. 6(a) and (b) shows the determined peak images. As shown in Fig. 4, a peak is properly produced at the OD position. On the other hand, a peak is also produced at the macula center (i.e., fovea) that often has similar peak amplitude to the peak at the OD center. This can be explained by similar brightness variation structure around the macula, where the image variation along the line segment crossing the macula center reaches the maximum, whereas that along the orthogonal line segment [similar to Lc and Lt in Fig. 1(b)] reaches the minimum. The only difference is that the OD center is brighter than the surrounding pixels, whereas the macula center is darker.

We, therefore, first classify the peaks into an OD category and a macula category, respectively. The classification is based on the image difference between the retinal image pixels at the peak center and those surrounding the peak center. The image difference is evaluated by two concentric circles as follows:

$\operatorname{Diff}(\mathrm{x}, \mathrm{y})=\frac{1}{N_{i}} \sum_{d=0}^{R_{1}} I(d)-\frac{1}{N_{o}} \sum_{d=0}^{R_{2}} I(d)$

where I refers to the retinal image under study and d denotes the distance between the peak and the surrounding retinal image pixels. $R_{1}$ and $R_{2}$ specify the radius of an inner concentric circle and an outer concentric circle where $\mathrm{R}_{2}$ is set at $2 \mathrm{R}_{1} \cdot \mathrm{N}_{i}$ and $\mathrm{N}_{o}$ denote the numbers of the retinal image pixels within the two concentric circles. In our system, we set $R_{1}$ at $(p-1) / 2$, where $p$ is the length of the line operator. The peak can, therefore, be classified to the OD or macula category, if the image difference is positive or negative, respectively.

Finally, we detect the OD by a score that combines both the peak amplitude and the image intensity difference that by itself is also a strong indicator of the OD

$\mathrm{S}(\mathrm{x}, \mathrm{y})=\mathrm{P}(\mathrm{x}, \mathrm{y})(\operatorname{Diff}(\mathrm{x}, \mathrm{y}) *(\operatorname{Diff}(\mathrm{x}, \mathrm{y})>0))$

Where $\mathrm{P}(\mathrm{x}, \mathrm{y})$ denotes the normalized peak image. The symbol * denotes dot product and $\operatorname{Diff}(\mathrm{x}$, $y)>0$ sets all retinal image pixels with a negative image difference to zero. The OD can, therefore, be detected by the peak in the OD category that has the maximum score. For the example retinal image in Fig. 1(a), Fig. 5(a) shows the score image determined by the peak image in Fig. 4(b). It should be noted that the image difference is evaluated only at the detected peaks in practical implementation. The score image in Fig. 5(a) (as well as in Fig. 5(b), 9, and 10) where the image difference is evaluated at every pixel is just for the illustration purpose. 


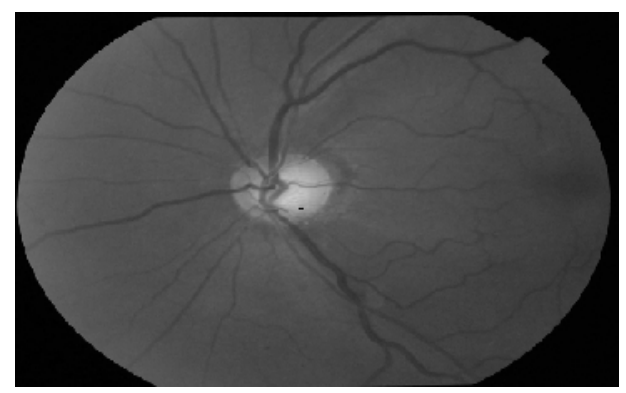

Fig.5. OD detection. Score image by (9) for OD detection.

\section{EXPERIMENTAL RESULTS}

The proposed algorithm has been implemented in MATLAB. The corresponding results are follows.

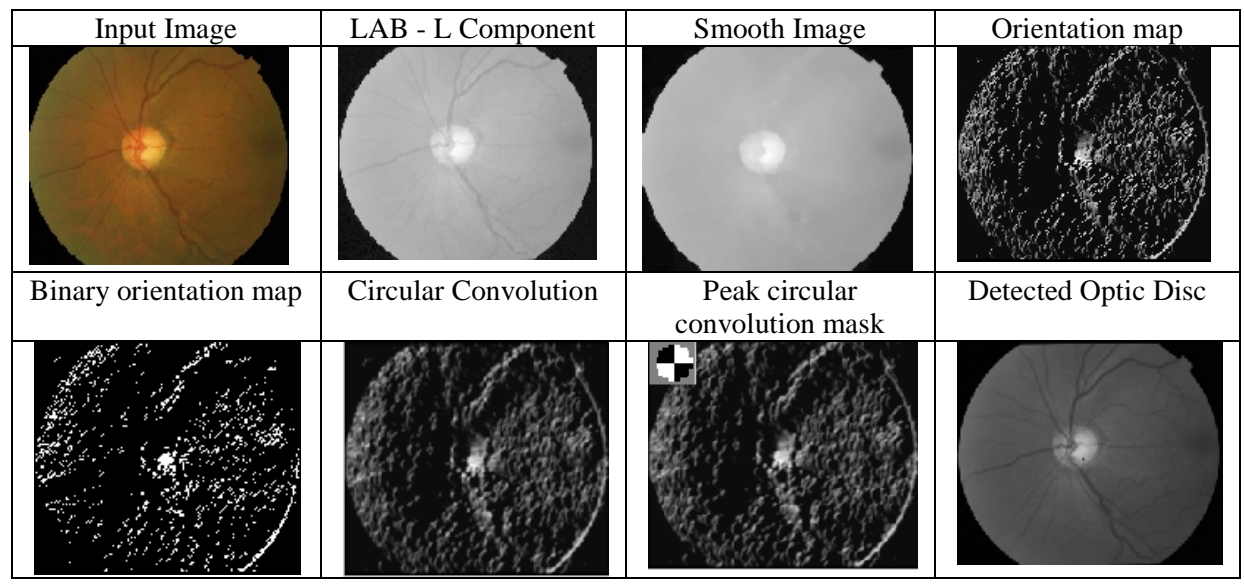

\section{CONCLUSION AND FUTURE WORK}

The paper presented a simple method for OD detection using Line operator filter. The proposed approach achieved better results as reported. An extension for this study could be as follows.

The image segmentation is the foundation for the retinal fundus images. Efficient algorithms for segmenting the region of interest would be the main objective of future work. Optic disc can be detected easily after segmentation. A myriad of Optic Disc detection methods are available. The method which is compatible with the segmentation algorithm should be used. Diseases like Glaucoma and Diabetic Retinopathy are in rise and Optic Disc is an important indicator of these diseases. Hence the new methodologies to detect Optic Disc and the efficient use of already existing methods are the interest of future work.

\section{ACKNOWLEDGMENTS}

The authors would like to acknowledge the faculty of Electronics and Communication Engineering department of Aalim Muhammed Salegh College of Engineering for their continuous support and encouragement and also acknowledge centre for research St.Joseph's College of Engineering, Chennai, Tamilnadu, India. 
International Journal of Advanced Information Technology (IJAIT) Vol. 2, No.4, August 2012

\section{REFERENCES}

[1] K. Akita and H. Kuga, "A computer method of understanding ocular fundus images," Pattern Recognit., vol. 15, no. 6, pp. 431-443, 1982.

[2] N. Patton, T. M. Aslam, T. MacGillivary, I. J. Deary, B. Dhillon, R. H. Eikelboom, K. Yogesan, and I. J. Constable, "Retinal image analysis:Concepts, applications and potential," Prog. Retin. Eye Res., vol. 25,no. 1, pp. 99-127, 2006.

[3] T. Walter, J. C. Klein, P. Massin, and A. Erginay, "A Contribution of Image Processing to the Diagnosis of Diabetic Retinopathy-Detection of Exudates in Color Fundus Images of the Human Retina,” IEEE Trans.Med. Imag., vol. 21, no. 10, pp. 1236-1243, Oct. 2002.

[4] Chrastek, M. Wolf, K. Donath, H. Niemann, D. Paulus, T. Hothorn, B. Lausen, R. Lammer, C. Y. Mardin, and G. Michelson, "Automated segmentation of the optic nerve head for diagnosis of glaucoma," Med.Image Anal., vol. 9, no. 4, pp. 297-314, 2005.

[5] A. D. Fleming, K. A. Goatman, S. Philip, J. A. Olson, and P. F. Sharp, "Automatic detection of retinal anatomy to assist diabetic retinopathy screening," Phys. Med. Biol., vol. 52, no. 2, pp. 331-345, 2007.

[6] A. Pinz, S. Bernogger, P. Datlinger, and A. Kruger, "Mapping the Human Retina," IEEE Trans.Med. Imag., vol. 17, no. 4, pp. 606-619, Aug. 1998.

[7] K.W. Tobin, E. Chaum, V. P. Govindasamy, and T. P. Karnowski, "Detection of anatomic structures in human retinal imagery," IEEE Trans. Med.Imag., vol. 26, no. 12, pp. 1729-1739, Dec. 2007.

[8] M. Niemeijer, M. D. Abramoff, and B. V. Ginneken, "Segmentation of the optic disc, macula and vascular arch in fundus photographs," IEEE Trans. Med. Imag., vol. 26, no. 1, pp. 116-127, Jan. 2007.

[9] W. Hsu, P. M. D. S. Pallawala, M. L. Lee, and K. A. Eong, "The Role of Domain Knowledge in the Detection of Retinal Hard Exudates,” in Proc.Int. Conf. Comp. Vis. Pattern Recognit., vol. 2, 2001, pp. 246-251.

[10] Z. B. Sbeh, L. D. Cohen, G. Mimoun, and G. Coscas, "A new approach of geodesic reconstruction for drusen segmentation in eye fundus images,” IEEE Trans. Med. Imag., vol. 20, no. 12, pp. 1321-1333, Dec. 2002.

[11] T. Walter and J. C. Klein, "Segmentation of color fundus images of the human retina: Detection of the optic disc and the vascular tree using morphological techniques," in Proc. Int. Symp. Med. Data Anal., 2001, pp. 282-287.

[12] H. Li and O. Chutatape, "Automatic location of optic disc in retinal images," in Proc. Int. Conf. Image, vol. 2, pp. 837-840, 2001.

[13] C. Sinthanayothina, J. F. Boycea, H. L. Cookb, and T. H. Williamsonb, "Automated localisation of the optic disc, fovea, and retinal blood vessels from digital colour fundus images," Br. J. Ophthalmol., vol. 83, pp. 902-910, 1999.

[14] S. Sekhar, W. Al-Nuaimy, and A. K. Nandi, "Automated localisation of retinal optic disk using Hough transform,” in Proc. Int. Symp. Biomed. Imag.: Nano Macro, 2008, pp. 1577-1580.

[15] A. Youssif, A. Z. Ghalwash, and A. Ghoneim, "Optic disc detection from normalized digital fundus images by means of a vessels' directionmatched filter,” IEEE Trans. Med. Imag., vol. 27, no. 1, pp. 11-18, Jan. 2008.

[16] A. Hoover and M. Goldbaum, "Locating the optic nerve in a netinal image using the fuzzy convergence of the blood vessels," IEEE Trans. Med. Imag., vol. 22, no. 8, pp. 951-958, Aug. 2003.

[17] M. Foracchia, E. Grisan, and A. Ruggeri, "Detection of optic disc in retinal images by means of a geometrical model of vessel structure,” IEEE Trans. Med. Imag., vol. 23, no. 10, pp. 1189-1195, Oct. 2004.

[18] F. Haar, "Automatic localization of the optic disc in digital colour images of the human retina," M.S. thesis, Utrecht University, Utrecht, The Netherlands, 2005.

[19] H. Li and O. Chutatape, "Automated feature extraction in color retinal images by a model based approach,” IEEE Trans. Biomed. Eng., vol. 51, no. 2, pp. 246-254, Feb. 2004.

[20] A. P. Rovira and E. Trucco, "Robust optic disc location via combination of weak detectors," in Proc. Int. Conf. IEEE Eng. Med. Bio. Soc., 2008, pp. 3542-3545.

[21] R. Zwiggelaar, S. M. Astley, C. R. M. Boggis, and C. J. Taylor, "Linear structures in mammographic images: Detection and classification,” IEEE Trans. Med. Imag., vol. 23, no. 9, pp. 1077-1086, Sep. 2004.

[22] E. Ricci and R. Perfetti, "Retinal blood vessel segmentation using line operators and support vector classification,” IEEE Trans. Med. Imag., vol. 26, no. 10, pp. 1357-1365, Oct. 2007. 
International Journal of Advanced Information Technology (IJAIT) Vol. 2, No.4, August 2012

[23] A. Osareh, M. Mirmehdi, B. Thomas, and R. Markham, "Comparison of colour spaces for optic disc localisation in retinal images," in Proc. Int. Conf. Pattern Recognit., vol. 1, 2002, pp. 743-746.

[24] C. Tomasi and R. Manduchi, "Bilateral Filtering for Gray and Color Images," in Proc. IEEE Int. Conf. Comp. Vis., 1998, pp. 839-846.

[25] W. Tasman and E. A. Jaeger, Duane's Ophthalmology, 15th ed. Baltimore, MD: Lippincott Williams \& Wilkins, 2009.

[26] T. Kauppi, V. Kalesnykiene, J. K. Kamarainen, L. Lensu, I. Sorri, H. Uusitalo, H. K“alvi“ainen, and J. Pietil"a, "DIARETDB0: Evaluation database and methodology for diabetic retinopathy algorithms," Tech. Rep., Lappeenranta Univ. Technol., Lappeenranta, Finland, 2006.

[27] T. Kauppi, V. Kalesnykiene, J. K. Kamarainen, L. Lensu, I. Sorri, H. Uusitalo, H. Klviinen, and J. Pietil, "DIARETDB1 diabetic retinopathy database and evaluation protocol," Tech. Rep., Lappeenranta Univ. Technol., Lappeenranta, Finland, 2007.

[28] J. J. Staal, M. D. Abramoff, M. Niemeijer, M. A. Viergever, and B. V. Ginneken, "Ridge based vessel segmentation in color images of the retina," IEEE Trans. Med. Imag., vol. 23, no. 4, pp. 501-509, Apr. 2004.

\section{Authors}

R.Murugan was awarded BE degree in ECE from Sethu Institute of Technology affiliated to Anna University Chennai and his ME degree in Embedded System Technologies from K.S.R.College of Engineering affiliated to Anna University Coimbatore, Currently he is doing his Ph.D in Anna University of Technology Chennai for the field of Medical Imaging. He has 5 years experience in teaching. He is working as Assistant Professor in AMS College of Engineering, Avadi-IAF, Chennai. He published one International Journal and presented five national conferences \& two international conference. His research interest includes medical Imaging and Signal Processing.

Dr.Reeba Korah was awarded BE degree in Electronics Engineering from Marathwada University, Maharashtra and ME degree in Mechatronics from Anna University, Chennai and Ph.d from Anna University,Chennai. She has 19 years experience in teaching. She is currently working as Professor in St.Joseph's College of Engineering, Chennai, Tamilnadu, India. She published many leading international \& national journals and she is guiding 12 research scholars her research interest include VLSI, Signal Processing, wireless Sensor Networks and Semiconductor Devices.
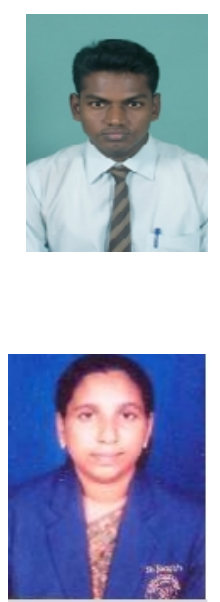